This item was submitted to Loughborough's Research Repository by the author.

Items in Figshare are protected by copyright, with all rights reserved, unless otherwise indicated.

\title{
The effect of active pharmaceutical ingredients on aerosol electrostatic charges from pressurized metered dose inhalers
}

PLEASE CITE THE PUBLISHED VERSION

http://dx.doi.org/10.1007/s11095-015-1674-6

\section{PUBLISHER}

(C) Springer

VERSION

SMUR (Submitted Manuscript Under Review)

\section{PUBLISHER STATEMENT}

This work is made available according to the conditions of the Creative Commons Attribution-NonCommercialNoDerivatives 4.0 International (CC BY-NC-ND 4.0) licence. Full details of this licence are available at: https://creativecommons.org/licenses/by-nc-nd/4.0/

\section{LICENCE}

CC BY-NC-ND 4.0

\section{REPOSITORY RECORD}

Chen, Yang, Paul M. Young, David F. Fletcher, Hak Kim Chan, Edward J. Long, David Lewis, Tanya Church, and Daniela Traini. 2019. "The Effect of Active Pharmaceutical Ingredients on Aerosol Electrostatic Charges from Pressurized Metered Dose Inhalers”. figshare. https://hdl.handle.net/2134/25339. 
The Effect of Active Pharmaceutical Ingredients on Aerosol Electrostatic Charges from Pressurized Metered Dose Inhalers

Yang Chen ${ }^{1}$, Paul M. Young ${ }^{1}$, David F. Fletcher ${ }^{2}$, Hak Kim Chan ${ }^{3}$, Edward Long ${ }^{4}$, David Lewis ${ }^{5}$, Tanya Church $^{5}$, Daniela Traini ${ }^{1 *}$

${ }^{1}$ Respiratory Technology, Woolcock Institute of Medical Research and Discipline of Pharmacology, Sydney Medical School, The University of Sydney, NSW 2037, Australia.

${ }^{2}$ School of Chemical and Biomolecular Engineering, The University of Sydney, NSW 2006, Australia.

${ }^{3}$ Advanced Drug Delivery Group, Faculty of Pharmacy (A15), University of Sydney Sydney, NSW 2006, Australia.

${ }^{4}$ Wolfson School of Mechanical and Manufacturing Engineering, Loughborough University, Loughborough, Leicestershire, LE11 3TU United Kingdom.

${ }^{5}$ Chiesi Ltd, Units T1 - T3, Bath Rd. Ind. Est., Chippenham, Wiltshire, SN14 0AB United Kingdom.

*To whom correspondence should be addressed

Daniela Traini

Tel: +61291140352

Email: Daniela.traini@sydney.edu.au

Keywords: electrostatics charges, aerosols, metered dose inhalers, APIs. 


\section{Introduction}

Aerosolised medication is often prescribed for the treatment of respiratory illness such as asthma and chronic obstructive pulmonary disease $(1,2)$. The pressurised metered dose inhaler (pMDI) is a system designed to generate an inhalable aerosol that accurately delivers the active pharmaceutical ingredients (APIs) to the respiratory tract (3). This occurs through the following five mechanisms: impaction, sedimentation, interception, diffusion and electrostatic deposition (4-6). Whilst the first four mechanisms have been extensively studied over the years (7-9) and are closely related to the aerosols physical characteristics, such as particle size and shape, it was not until recently that electrostatics forces became an area of interest in pulmonary drug delivery (10).

Most pharmaceutical ingredients are dielectric materials and highly susceptible to electrostatic charge generation and accumulation, through contact/friction charging between particles and material surfaces (11-13). Although it is well recognised that electrostatic charges can be a nuisance during manufacture and handling of pharmaceutical powders (by promoting agglomeration, segregation and adhesion, especially when fine particles are involved $(14,15)$, research studies using theoretical predictions (16-19), in vitro lung models $(20,21)$, in vivo animal and human subjects (22-26) have all suggested that electrostatic force on charged particles can significantly influence the aerosol performance for pulmonary drug delivery.

The deposition of charged particle in the respiratory tract is described by two general mechanisms: space and image charge. The former space charge refers to the natural 
repulsive force created by the electron cloud on the charged particles, especially when aerosol are condensed (27). Therefore, space charges play an important role during pMDI plume formation and consequently deposition on the pharyngeal tracheal region (28). The latter, image charge, predominantly influences the downstream aerosol deposition through induction of image charges with opposite polarity on nearby surfaces, for example the airway wall, hence promoting electrostatic deposition in the lung $(10,27)$.

When a pMDI is actuated, the pressurised mixture of hydrofluoroalkane (HFA) propellant and drug (solubilised or suspended) with or without co-solvents/excipients is exposed to the atmospheric pressure. Consequently, the rapid transition of propellant into its gaseous state aerosolises the APIs carried by the HFA. Flash boiling, cavitation and evaporation during the atomisation process creates an interaction between the liquid, solid and gas components of the formulation, providing contact surfaces for triboelectrification. Therefore, factors that contribute to electrostatic charge generation for pMDI aerosols are often related to the physical and chemical properties of the device, including the material used for the actuator, the design of the orifice nozzles and chemical structure of the drug and excipients used in the formulation. In previous studies, different actuator materials and nozzle designs were selected from the triboelectric series and assessed for their influence on the resultant electrostatic properties $(28,29)$. It was found that the net charge profiles obtained with a formulation containing no drug and low co-solvent (ethanol 1\%) emitted from a cone nozzle design followed the triboelectric series (29) . However, when an active pharmaceutical ingredient (API; beclomethasone dipropionate) was introduced into the formulation, no 'trend' was found and the net charge profiles 
changed between different actuator materials (29). These results suggested that the API in a pMDI formulation has a strong influence on the overall electrostatic properties of the generated aerosol plume.

In nature, the driving mechanism for triboelectrification is the materials' work function. It is an indication of the materials' surface property and refers to the energy required to remove electrons from a solid to an immediate point outside the solid (in a vacuum) (30). In metals, the valance band is filled with electrons up to the Fermi level, which overlap with the conduction band. Therefore, electron can move freely within a metal and the work function is the energy difference between the Fermi levels and the vacuum. For insulators, the Fermi level lies in the large band gap that exits between the valance and the conduction band, indicating that no electrons are present in the conduction band and it therefore has a much higher work function energy (31-33). During contact charging, two materials come in contact and electrons will transfer from lower to higher work function in attempt to reach thermodynamic equilibrium (34). After separation, the material that gains electron(s) will charge negatively and the material that loses electron(s) will charge positively. However, the work function is strongly influenced by the materials' surface conditions, including contamination of other atoms/molecule, surface reaction (oxidation, ionisation), surface structures, as well as environmental factors such as humidity, especially for dielectric materials used in pharmaceutical aerosols (35).

In pMDIs, drug particles are generated through complex phase transitions and chemical interactions between the propellant, co-solvent/excipients and the APIs. 
Thus it is difficult to predict the electrostatic potential for pMDI aerosols and current studies can only rely on in vitro screening analysis for individual factors that could contribute to aerosol electrostatics. Therefore, this study focused on investigating four solution-based pMDIs with different APIs (beclomethasone dipropionate, budesonide, salbutamol base and ipratropium bromide, respectively) to elucidate how active drugs can influence aerosol electrostatic charge, with both insulating and conducting actuator materials.

\section{$2 \quad$ Materials and Methods}

\section{$2.1 \quad$ Materials}

Five active pharmaceutical ingredients (APIs), comprising beclomethasone dipropionate, budesonide, flunisolide, salbutamol base and ipratropium bromide were chosen as model drugs (Figure 1) and supplied by Chiesi Farmaceutici S.p.A (Parma, Italy). Standard aluminium pMDI canisters (C128P, ID214, Batch 1002043-3, 18 ml brim capacity) were obtained from Presspart Manufacturing Ltd (Lancashire, UK) and fitted with $50 \mu 1$ metering valves (ID201, batch BK0313029, Bespak Europe Ltd, Norfolk, UK). The propellant 1,1,1,2-Tetrafluoroethane (HFA 134a) was provided by Solvay Chemicals (Brussels, Belgium), and all other analytical grade chemicals, used as received, were purchased from Sigma-Aldrich Pty Ltd (Castle Hill, Australia). Water used through out the study was purified by reverse osmosis (Milli-Q, Sydney, Australia). 


\subsection{Sample Preparation}

Five solution-based pMDIs containing different API ingredients were prepared using pressure filling technique according to Table 1 . The required quantity of individual drugs were accurately weighed and dissolved in co-solvent ethanol $(14.9 \% \mathrm{w} / \mathrm{w})$ into aluminum canisters. Each canister was immediately crimped with the metering valve and pressure filled with propellant HFA 134a using a Pamasol P2016 laboratory crimp and filling plant (Pamasol Willi Maäden AG, Pfaffikon, SZ). Solubility of the drug was confirmed visually using glass containers (Saint Gobain, France). All canisters were stored at ambient temperature for 24 hours prior to testing.

\subsection{Actuators manufacture}

The pMDI actuator blocks were manufactured using three different materials including nylon, polytetrafluoroethylene (PTFE) (both from Ensinger GmbH, Nufringen, Germany) and aluminum (Aalco Metals Ltd, Cobham, UK); representing positive, negative and conducting triboelectric materials, respectively (36). The nozzle design with a nominal orifice diameter of $0.3 \mathrm{~mm}$ and cone outer shape was selected to represent the most commonly utilized geometry in commercial pMDI actuators and manufactured with Siemens $N X$ software using high-speed-steel cutting tools. Orifice diameters were confirmed using microscope and MediaCybernetics Image-Pro software, with dimensional accuracy up to $\pm 0.01 \mathrm{~mm}$. All actuator blocks were washed and sonicated with purified water and ethanol prior to first use. Airdrying was used instead of heat drying to prevent changes to the orifice diameter. Adaptors to house the actuator block of the pMDI were custom designed using 
computer aided design (ANSYS DesignModeler, release 13, ANSYS Inc, PA, USA) and built in acrylonitrile butadiene styrene (ABS) using a 3D printer (Dimension Elite, MN, USA).

2.4 Measurements of aerosols electrostatic charge

A modified 13 stage electrical low-pressure impactor (ELPI ${ }^{\mathrm{TM}}$, Dekati Ltd, Finland), without the corona charger, was used to measure the aerosol electrostatic charge distribution, as described previously $(29,37)$. The pMDI was shaken for $10 \mathrm{~s}$ and primed to waste twice using a commercial actuator before being fitted to the actuator block with the custom built adaptor. The airflow through the ELPI was set at 30 L/min using Sogevac® model SV25 vacuum pump (Leybold, France) and a calibrated Copley ${ }^{\circledR}$ model 4000 flow meter (Nottingham, UK). The pMDI unit was connected to the ELPI via United States Pharmacopeia (USP) induction port and baseline zeroed after the electrometer readings were stabilized. Five single actuations from each pMDI formulation were dispersed into the ELPI cumulatively, with $30 \mathrm{~s}$ delay between each dose. The aerosol charges were measured and current recorded using the ELPI-VI 4.0 software (Dekati Ltd, Finland) as femto amps per second (fA/s) and then converted to charge data during analysis. All experiments were randomized and performed in triplicate under standard laboratory conditions (temperature $\sim 25^{\circ} \mathrm{C}$ and relative humidity $\sim 40-50 \%$ ).

2.5 High performance liquid chromatography 
The drug captured on the actuator block, adaptor, USP induction port and impactor stages was recovered using rinsing solution (Table 2) specific for each API and quantified chemically using a Shimadzu prominence UFLC system equipped with a SPD-20A UV-vis detector, LC-20AT solvent delivery unit, SIL-20A HT autosampler (Shimadzu Corporation, Japan). Chromatographic conditions for each API formulation are summarized in Table 2. Fresh drug standards were prepared in rinsing solution and all mobile phase solutions were filtered through $0.45 \mu \mathrm{m}$ filters and degassed by ultra-sonication for $10 \mathrm{~min}$. The HPLC method was validated for all APIs throughout the concentration range of $0.1-100 \mu \mathrm{g} / \mathrm{mL}$.

\subsection{Data Analysis}

Aerosol electrostatic charge data for each pMDI formulation were derived from electric current results obtained from the ELPI. The net charge was calculated as the total charge from the 13 stages of the impactor and shown as the mean for the three experiments. The mass recoveries of individual APIs were analyzed as the total mass of five cumulative actuations. Total ex-valve dose, throat USP deposition and fine particle fraction $(<6.66 \mu \mathrm{m})$ were calculated and expressed as a percentage of the targeted dose (5 accumulative shot of $50 \mu$ g per shot, equivalent to $250 \mu \mathrm{g}$ ). Mass median aerodynamic diameter (MMAD) was calculated assuming linearity between $84-16 \%$ of the cumulative mass undersize lognormal distribution and the geometric standard deviation (GSD) was determined as $\left(\mathrm{d}_{0.84} / \mathrm{d}_{0.16}\right)^{1 / 2}$. Charge to mass ratio was analysed as total charge and mass of three replicate experiments. Two sample Student t-test (heteroscedastic) and one-way ANOVA (unstacked) analysis was performed 
using STATPlus ${ }^{\circledR}$ statistics software package (AnalystSoft Inc, VA, USA).

Significant difference was based $p<0.05$.

\section{Results and Discussion}

The influence of different active pharmaceutical ingredients, formulated as solution pMDIs, on the aerosol performance and electrostatic charge profiles have been investigated in this study and results are discussed below.

3.1 The effect of APIs on overall aerosol net charge using different actuator materials

The net charge of five different APIs and three different actuator materials were calculated as the total charge derived from the 13 stages of the ELPI. The mean of the three replicate experiments are shown in Figure 2. Actuator material Nylon and PTFE were selected to represents the extreme of the triboelectric series, with Nylon being positive and PTFE negative charged materials, respectively. Aluminium is ranked close to neutral and was selected as conducting material for comparison (38).

In general, all three actuator materials showed the same net charge trend across the five active drug ingredients, with beclomethasone dipropionate (BDP), budesonide (BUD) and flunisolide (FS) showing positive net charges, while salbutamol base (SB) and ipratropium bromide (IPBr) having negative net charges. Statistical analyses using one-way ANOVA showed no significant differences between nylon, aluminium and PTFE actuators, when the same API was used, but significant difference across different APIs when the same actuators was used (one way ANOVA, $p<0.05$ ). This is 
consistent with a previous study where the addition of active drug ingredient in a pMDI formulation diminished the actuator materials' triboelectric effect on aerosol charges (39).

For both Nylon and PTFE actuators, which are insulating thermoplastics with distinctively different static charging trends, BDP, BUD and FS showed positive charges, ranging from $134.78 \pm 127.29$ pC (BUD with Nylon actuator) to $332.74 \pm$ 86.74 pC (BUD with PTFE actuator), but no significant differences (Student t test, Figure 2). These results suggest that the API may have a dominant effect on the measured aerosol electrostatic charge (Figure 2). BDP, BUD and FS are all corticosteroids with very similar molecular structures (Figure 1). They all contain electronegative atoms, such as oxygen and fluorine, capable of dipole-dipole attraction and hydrogen bonding. The interaction between the corticosteroids, HFA 134a and co-solvent ethanol could result in a change of work function for the final aerosol particles, and similar functional groups on the BDP, BUD and FS molecules could produce comparable work functions for the three drugs, inducing similar contact charging profiles with the actuator material surfaces. Additionally, it is important to recognize that triboelectrification between insulators are complex. In theory, electrons would flow from a material with low work function to the one with higher work function. From the net charge results, BDP, BUD and FS all have dominantly positive charge polarity, indicating an electron transfer from the aerosol to the actuator material (Figure 2, Nylon and PTFE). A possible explanation for such observation could be the presence in the formulation of $15 \%$ co-solvent ethanol. A previous study has shown that ethanol reduced the electronegativity of HFA 134 propellant and shifted the net charge for a pMDI aerosol toward positive/neutrality 
(39). At the same time, interaction between co-solvent, drug and propellant within the formulation could potentially reduce the dielectric properties of the aerosol, hence reducing the work function energy. Lower work function allows the material to lose electrons, which is reflected in the net charge results (Figure 2).

When BDP, BUD and FS pMDI formulations were used with the aluminum conducting metal actuator, results showed almost neutral net charge profiles, with significantly lower magnitude compared with nylon and PTFE, with an average less than $50 \mathrm{pC}$ (Figure 2, Aluminum). These results might be due to the fact that the work function of these corticosteroids formulations are close to the work function of aluminum, hence electron transfer between the aerosols and material surface is limited. Meanwhile, the conducting property of aluminum allows free movement of electrons within the solid body, hence could form a negative electron cloud on the surface of the material and potentially neutralize the positive charges on the aerosol particles after contact charging.

In comparison, significant differences in net charges were observed between SB and $\mathrm{IPBr}$ (Figure 2) with the same actuator material (Student t-test, $p<0.05$ ). SB showed a negative charge profile for all three actuators, with the highest magnitude shown with PTFE at $-930.32 \pm 300.25 \mathrm{pC}$ (Figure 2). SB is a short acting $\beta 2$-adrenergic receptor agonist. Its molecular structure contains three hydroxyl groups. It is hypothesized that the strong electronegativity of the HFA 134a propellant may attract hydrogen ions forcing the hydroxyl groups to become hydroxide anions and carry negative charges. Therefore, the overall net charge for SB is negative (Figure 2). 
$\mathrm{IPBr}$ also demonstrated negative charge polarity with all actuator materials tested, but with a smaller magnitude, at an average of $-334.52 \pm 90.73 \mathrm{pC}$. IPBr is an anticholinergic drug, with a permanently positively charged ammonium cation and negatively charged bromide ion. The bipolar nature of IPBr could cause potential neutralization of the electrostatic charges generated following triboelectrification of the aerosol droplets with the actuator materials, hence generating a reduced net charge magnitude (Figure 2).

In is interesting to note that the conducting actuator aluminum did not show significant difference in net charges with SB and IPBr, compared with nylon and PTFE actuators. Probably, due to the functional groups and ions present, the conductivity of SB and IPBr increases and the work function can be reduced. This should cause electrons to flow from the aerosols to the material surfaces, in contrast with the observed results (Figure 2). These results could be related to the presence of ionic groups within the formulation, depended on the $\mathrm{pH}$ of the HFA/ethanol solution. However, since PMDIs are pressurised systems, it is difficult to determine the actual physiochemical properties of the mixture inside the canisters and therefore hard to predict the possible downstream effects of molecular functional groups on aerosol electrostatics. Future studies will investigate this aspect of the project.

3.2 The effect of APIs on aerosol performance using different actuator materials

Aerosol electrostatic results have shown that the APIs have a dominant effect on pMDI aerosol charge profiles. Since aerosol performance can be influence by both 
drug formulations and device design, it is important to investigate the aerodynamic properties for the pMDIs and examine the correlation between particle characteristics and static charges.

Cumulative particle size distribution plots were calculated from the cumulative mass under-size for each individual API and are shown in Figure 3. Statistical analyses using one-way ANOVA showed no significant differences between drugs for the same material, as well as the same drug with different materials, indicating different drug ingredients have no influence on particle size distribution for the pMDI formulations. Significant differences in MMADs were observed across the different APIs for all three actuator materials (one way ANOVA); with BDP $0.76 \pm 0.01 \mu \mathrm{m}$, BUD $0.91 \pm 0.07 \mu \mathrm{m}$, FS $0.89 \pm 0.00 \mu \mathrm{m}$, SB $0.70 \pm 0.02 \mu \mathrm{m}$ and IPBr $0.95 \pm 0.02$ $\mu \mathrm{m}$, respectively. A general mean GSD value at $2.31 \pm 0.29 \mu \mathrm{m}$ indicated all pMDI formulations were poly-dispersed.

Furthermore, total ex-valve dose, throat USP deposition and fine particle fractions less than $6.66 \mu \mathrm{m}$ were analyzed, based on the percentage of the designed target dose and shown in Figures 4, 5 and 6. Statistical analyses indicated significant differences in total ex-valve dose between different APIs using nylon, aluminum and PTFE actuators. Higher emitted dose were shown with BDP and FS for all three materials, compared with the other three APIs (Figure 4), suggesting less drug retention in the device. However, the emitted dose for all drugs did not correlate with the static charge profiles, which could be due to reduced charge magnitude (Figure 2). 
Drugs collected in the USP throat for all API formulations are shown in Figure 5. One-way ANOVA showed statistically significant difference across different APIs for the same actuator material; SB had the smallest USP deposition comparedwith the other drugs. This is an interesting observation, as SB had the most negative net charge profile. It was hypothesized that unipolar dominant charged aerosols, where plume expansion is possible due to the presence of space charges, could have increased the deposition to the throat region, but this was not reflected in the SB results. A possible explanation could be linked to the density of the aerosol droplets. From the molecule structure of SB (Figure 1), it is know that SB had the lowest density among the five APIs studied. This generates smaller particles after atomization, supported by the small MMAD of SB $(0.70 \pm 0.02 \mu \mathrm{m})$. Although small size particles tends to charge more negatively (40), it could also travel at a higher velocity, limiting the time for the plume expansion effects due to space charge, reducing throat deposition (Figure 5).

The fine particle fraction of particles less than $6.66 \mu \mathrm{m}$ was calculated based on the drug mass recovered from the ELPI impactor stages and is shown as a percentage of the target dose in Figure 6. This is a representation of the respiratory fraction of the pMDI formulation that is suitable for lung deposition. No significant differences were found in FPF for the same drug with different actuator materials. However, significant higher fine particle fractions were found with BDP using nylon $(36.55 \% \pm 5.91)$ and PTFE (34.34\% \pm 7.09) actuators, respectively, compared with aluminum and other pMDI formulations.

Although these results show that larger amount of positive charge particles could 
result in a higher fine particle fraction, the same results were not observed with other APIs and actuator materials (Figure 6). In general, there was no clear relationship between the electrostatic charges and aerosol performance, using different APIs.

3.3 The effect of APIs on charge to mass ratio using the aluminum actuator material

It is important to note that while there is no clear trend between aerosols charge and aerosol performance, the elementary charge of each particle can be important in understanding the involvement of charges in particle behaviours. To investigate this aspect, mass to charge ratios were calculated by dividing the net charge with the total mass recovery from the impactor. Results are shown in Table 3.

Similar trends as the net charge have been observed with the charge to mass ratio. Corticosteroids BDP, BUD and FS had positive elementary charges, with small magnitudes, ranging from $0.65 \pm 0.30 \mathrm{pC} / \mu \mathrm{g}$ (BUD with aluminium) to $29.21 \pm 8.78$ $\mathrm{pC} / \mu \mathrm{g}$ (BUD with PTFE) (Table 3), respectively. SB particles carried the most negative charges, especially with PTFE actuator $(-94.57 \pm 30.45 \mathrm{pC} / \mu \mathrm{g})$, which is equivalent to an elementary charge of $-31.04 \pm 12.59 \mathrm{pC}$ per particle. This amount of charge per particle is the highest among all five APIs studied, but is low compared with previous studies. Melandri et al. has shown that mono-dispersed particles could increase deposition when particles carried about 200 elementary charges per particle (22, 41); where Yu and Chandra found a threshold of 50 elementary charge per particle was required to alter deposition of $1 \mu \mathrm{m}$ particles (17). Both these studies have demonstrated deposition changes with unipolar charges and mono-dispersed 
particles. However, the dielectric properties of inhalation pharmaceutical powder show the aerosol will be dominated by bipolar charges, and the GSD value obtained from this study also shows particles are poly-dispersed. Therefore, no clear trends were observed between aerosol electrostatics and aerosol depositions for different APIs. However, it is significant that the use of drug ingredients in pMDI formulation is the determinant in aerosol charge polarity.

\section{Conclusions}

The API present in a pMDI has a dominant effect on the electrostatic properties of the formulation, overcoming the charge effect arising from the actuator materials. The presence of ionic functional groups in a drug molecule structure significantly influences the polarity of the generated electrostatic charges. In this study, no specific trend was observed between the deposition patterns and aerosol electrostatic charge profiles for the five API pMDIs formulations. However, results have shown that the electrostatic charges for a solution-based pMDI could be related to the interactions of the chemical ingredients and change in the work function for the overall formulation.

\section{$5 \quad$ References}

1. Global Initiative for Asthma Report: Global Strategy for Asthma Management and Prevention. 2014 (Available from http://www.ginasthma.org; accessed on 18 Nov, 2014).

2. Global Initiative for Chronic Obstructive Lung Disease (GOLD): Global Strategy for the Diagnosis, Management and Prevention of COPD. 2014 (Available from: http://www.goldcopd.org/; accessed on 17 Nov, 2014).

3. Newman SP. Principles of metered-dose inhaler design. Respir Care. 2005;50(9):1177-90.

4. Suarez S, Hickey AJ. Drug properties affecting aerosol behavior. Respir Care. 2000;45(6):652-66. 
5. O'Doherty M, Miller R. Aerosols for therapy and diagnosis. European Journal of Nuclear Medicine. 1993;20(12):1201-13.

6. Hinds WC. Aerosol technology: properties, behavior, and measurement of airborne particles. New York, Wiley-Interscience, 1982442 p. 1982;1.

7. Davies CN, Muir DC. Deposition of Inhaled Particles in Human Lungs. Nature. 1966 07/02/print;211(5044):90-1.

8. Heyder J. Particle transport onto human airway surfaces. European Journal of respiratory diseases Supplement. 1981;119:29-50.

9. Lourenco RV, Cotromanes E. Clinical aerosols: I. Characterization of aerosols and their diagnostic uses. Archives of Internal Medicine. 1982;142(12):2163-72.

10. Kwok PCL, Chan HK. Electrostatics of pharmaceutical inhalation aerosols. J Pharm Pharmacol. 2009 Dec;61(12):1587-99. PubMed PMID: ISI:000281136700002. 11. Bailey AG. Electrostatic Phenomena during Powder Handling. Powder Technology. 1984;37(Jan-):71-85. PubMed PMID: ISI:A1984SC64500007.

12. Bailey AG. Charging of Solids and Powders. Journal of Electrostatics. 1993 May;30:167-80. PubMed PMID: ISI:A1993LB81100017.

13. Bailey A. Electrostatic phenomena during powder handling. Powder Technology. 1984;37(1):71-85.

14. Rowley G. Quantifying electrostatic interactions in pharmaceutical solid systems. International journal of pharmaceutics. 2001;227(1):47-55.

15. Pu Y, Mazumder M, Cooney C. Effects of electrostatic charging on pharmaceutical powder blending homogeneity. J Pharm Sci-Us. 2009;98(7):2412-21. 16. Ingham DB. Precipitation of Charged-Particles in Human Airways. J Aerosol Sci. 1981;12(2):131-5. PubMed PMID: ISI:A1981LN21400005.

17. Yu CP, Chandra K. Precipitation of Submicron Charged-Particles in Human Lung Airways. B Math Biol. 1977;39(4):471-8. PubMed PMID:

ISI:A1977DL62700007.

18. Diu CK, Yu CP. Deposition from Charged Aerosol Flows through a Pipe Bend. J Aerosol Sci. 1980;11(4):397-402. PubMed PMID: ISI:A1980KM94500007. 19. Thiagarajan V, Yu CP. Sedimentation from Charged Aerosol Flows in Parallel-Plate and Cylindrical Channels. J Aerosol Sci. 1979;10(4):405-10. PubMed PMID: ISI:A1979HT24200007.

20. Chan TL, Yu CP. Charge Effects on Particle Deposition in the Human Tracheobronchial Tree. Ann Occup Hyg. 1982;26(1-4):65-75. PubMed PMID: ISI:A1982PT13100006.

21. Saini D, Gunamgari J, Zulaloglu C, Sims RA, Mazumder MK. Effect of electrostatic charge and size distributions on respirable aerosol deposition in lung model. Ieee Ind Applic Soc. 2004:948-52. PubMed PMID: ISI:000225148600137. 22. Melandri C, Tarroni G, Prodi V, Dezaiacomo T, Formignani M, Lombardi CC. Deposition of Charged-Particles in the Human Airways. J Aerosol Sci. 1983;14(5):657-69. PubMed PMID: ISI:A1983RT47400008.

23. Tarroni G, Melandri C, Prodi V, Dezaiacomo T, Formignani M, Bassi P. An Indication on the Biological Variability of Aerosol Total Deposition in Humans. Am Ind Hyg Assoc J. 1980;41(11):826-31. PubMed PMID: ISI:A1980KN75500008.

24. Ferin J, Mercer TT, Leach LJ. The Effect of Aerosol Charge on the Deposition and Clearance of Tio2 Particles in Rats. Environ Res. 1983;31(1):148-51. PubMed PMID: ISI:A1983QR93200013.

25. Fraser DA. Deposition of Unipolar Charged Particles in Lungs of Animals. Arch Environ Health. 1966;13(2):152-\&. PubMed PMID: ISI:A19668080900003. 
26. Vincent JH, Johnston WB, Jones AD, Johnston AM. Static Electrification of Airborne Asbestos - a Study of Its Causes, Assessment and Effects on Deposition in the Lungs of Rats. Am Ind Hyg Assoc J. 1981;42(10):711-21. PubMed PMID: ISI:A1981MK58000004.

27. Finlay WH. The mechanics of inhaled pharmaceutical aerosols: an introduction: Academic Press; 2001.

28. Chen Y, Traini D, Fletcher DF, Chan HK, Lewis DA, Church T, et al. The Effect of Pressurized Metered Dose Inhaler (pMDI) Actuator Nozzle Design on Triboelectrification and Aerosol Deposition. RDD Europe 2013 (2013). 2013;2:43944.

29. Chen Y, Young PM, Fletcher DF, Chan HK, Long E, Lewis D, et al. The Influence of Actuator Materials and Nozzle Designs on Electrostatic Charge of Pressurised Metered Dose Inhaler (pMDI) Formulations. Pharm Res. 2014;31(5):1325-37.

30. Hangsubcharoen M, Yan ES, Yoon RH, Luttrell GH. Evaluation of the Charging Characteristics of Particles in Triboelectrostatic Separation.

31. Singleton J. Band theory and electronic properties of solids: Oxford Univ. Press; 2001.

32. Bailey AG. The charging of insulator surfaces. Journal of Electrostatics. 2001;51:82-90.

33. Matsusaka S, Masuda H. Electrostatics of particles. Advanced Powder Technology. 2003;14(2):143-66.

34. Schlaf R. Tutorial on Work Function. USF Surface Science Laboratory.

35. Hoe S, Traini D, Chan H-K, Young PM. Measuring charge and mass distributions in dry powder inhalers using the electrical Next Generation Impactor (eNGI). European Journal of Pharmaceutical Sciences. 2009;38(2):88-94.

36. Diaz A, Felix-Navarro R. A semi-quantitative tribo-electric series for polymeric materials: the influence of chemical structure and properties. Journal of Electrostatics. 2004;62(4):277-90.

37. Kwok PCL, Glover W, Chan HK. Electrostatic charge characteristics of aerosols produced from metered dose inhalers. J Pharm Sci-Us. 2005

Dec;94(12):2789-99. PubMed PMID: ISI:000233733300018.

38. Diaz AF, Felix-Navarro RM. A semi-quantitative tribo-electric series for polymeric materials: the influence of chemical structure and properties. Journal of Electrostatics. 2004 Nov;62(4):277-90. PubMed PMID: ISI:000224503900003. English.

39. Chen Y, Young P, Fletcher D, Chan H, Long E, Lewis D, et al. The Influence of Actuator Materials and Nozzle Designs on Electrostatic Charge of Pressurised Metered Dose Inhaler (pMDI) Formulations. Pharm Res. 2014 2014/05/01;31(5):1325-37. English.

40. Lacks DJ, Levandovsky A. Effect of particle size distribution on the polarity of triboelectric charging in granular insulator systems. Journal of electrostatics. 2007;65(2):107-12.

41. Melandri C, Prodi V, Tarroni G, Formignani M, Bompane GF, Dezaiacomo T, et al. Deposition of Unipolarly Charged-Particles in Human Respiratory-Tract. Health Phys. 1977;33(3):273-. PubMed PMID: ISI:A1977EA33600022. 
6 Tables and Figures 
Table 1: Formulation details for the solution based pMDIs with different APIs.

\begin{tabular}{ccccc} 
APIs & Target Dose $(\boldsymbol{\mu g})$ & Drug $(\% \mathbf{w} / \mathbf{w})$ & Ethanol (\% w/w) & HFA 134a (\% w/w) \\
\hline BDP & 50 & 0.1 & 14.9 & 85 \\
BUD & 50 & 0.1 & 14.9 & 85 \\
FS & 50 & 0.1 & 14.9 & 85 \\
SB & 50 & 0.1 & 14.9 & 85 \\
IPBr & 50 & 0.1 & & 85 \\
\hline
\end{tabular}

BDP: Beclomethasone Dipropionate; BUD: Budesonide; FS: Flunisolide; SB: Salbutamol Base; IPBr: Ipratropium Bromide 
Table 2: Chromatographic conditions for the chemical assay.

\begin{tabular}{|c|c|c|c|c|c|c|}
\hline Formulations & $\begin{array}{c}\text { Rising } \\
\text { solution }\end{array}$ & Mobile Phase (v/v) & Column & $\begin{array}{l}\text { Flow Rate } \\
(\mathrm{ml} / \mathrm{min})\end{array}$ & $\begin{array}{c}\text { Injection } \\
\text { Volume } \\
(\mu \mathrm{l})\end{array}$ & $\begin{array}{c}\text { UV } \\
\text { Detection }\end{array}$ \\
\hline \multirow{2}{*}{ BDP } & 80\% Methanol & $68 \%$ Methanol & Waters & & & \\
\hline & $20 \% \mathrm{H} 2 \mathrm{O}$ & $\begin{array}{l}32 \% 0.05 \% \text { w/v Ammonia Acetate aqueous } \\
\text { solution }\end{array}$ & $\begin{array}{l}\text { Novapak }{ }^{\circledR} \\
\quad \text { C18 }\end{array}$ & 1 & 100 & $240 \mathrm{~nm}$ \\
\hline \multirow[t]{2}{*}{ BUD } & 80\% Methanol & 60\% Methanol & Waters & & & \\
\hline & $20 \% \mathrm{H} 2 \mathrm{O}$ & $40 \%$ deionized water & $\begin{array}{l}\text { Novapak }{ }^{\circledR} \\
\quad \text { C18 }\end{array}$ & 1 & 100 & 243nm \\
\hline \multirow[t]{2}{*}{ FS } & 80\% Ethanol & 35\% Acetonitrile & Waters & & & \\
\hline & $20 \% \mathrm{H} 2 \mathrm{O}$ & $65 \% 1 \% \mathrm{v} / \mathrm{v}$ acetic acid solution & $\begin{array}{l}\text { Bondapak }{ }^{\circledR} \\
\quad \text { C18 }\end{array}$ & 2 & 50 & $254 \mathrm{~nm}$ \\
\hline \multirow[t]{2}{*}{ SB } & 80\% Methanol & 60\% Methanol & Waters & & & \\
\hline & $20 \% \mathrm{H} 2 \mathrm{O}$ & $40 \% 0.1 \mathrm{w} / \mathrm{v}$ SDS aqueous solution & $\begin{array}{l}\text { Novapak }{ }^{\circledR} \\
\text { C18 }\end{array}$ & 1.5 & 100 & $276 \mathrm{~nm}$ \\
\hline \multirow[t]{2}{*}{ IPBr } & $100 \% \mathrm{H} 2 \mathrm{O}$ & 20\% Acetonitrile & Waters & & & \\
\hline & & 80\% Sodium phosphate Buffer pH4 & $\begin{array}{l}\text { Novapak }{ }^{\circledR} \\
\text { C18 }\end{array}$ & 1 & 100 & 210nm \\
\hline
\end{tabular}

BDP: Beclomethasone Dipropionate; BUD: Budesonide; FS: Flunisolide; SB: Salbutamol Base; IPBr: Ipratropium Bromide

All mobile phases were filtrated through a $0.45 \mu \mathrm{m}$ filter prior to HPLC use 
Table 3: Total charge per mass (pC/ $\mu \mathrm{g})$ of the APIs, $(\mathrm{n}=3, \pm \mathrm{SD})$

\begin{tabular}{cccc}
\hline API & Nylon $(\mathbf{p C} / \mu \mathbf{g} \pm \mathbf{S D})$ & Aluminium $\mathbf{( p C / \mu g} \pm \mathbf{S D})$ & $\mathbf{P T F E}(\mathbf{p C} / \boldsymbol{\mu g} \pm \mathbf{S D})$ \\
BDP & $11.63 \pm 3.37$ & $3.05 \pm 1.69$ & $18.48 \pm 17.09$ \\
BUD & $11.10 \pm 10.58$ & $0.65 \pm 0.30$ & $29.21 \pm 8.78$ \\
FS & $18.19 \pm 5.98$ & $0.81 \pm 0.83$ & $21.55 \pm 3.89$ \\
SB & $-73.63 \pm 25.28$ & $-35.46 \pm 7.54$ & $-94.57 \pm 30.45$ \\
IPBr & $-38.23 \pm 6.24$ & $-26.23 \pm 6.20$ & $-28.38 \pm 25.05$ \\
\hline
\end{tabular}




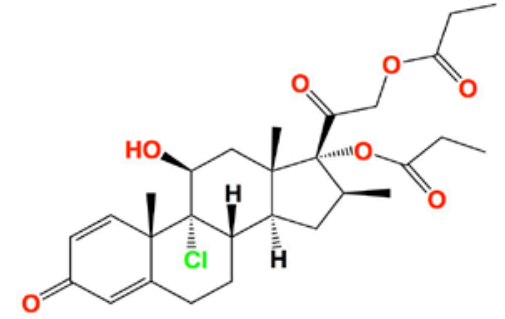

Beclomethasone Dipropionate<smiles>CC(C)(C)NCC(O)c1ccc(O)c(CO)c1</smiles>

Salbutamol Base

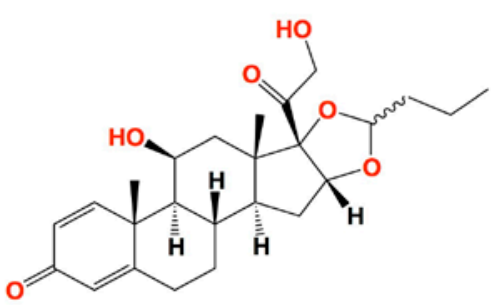

Budesonide

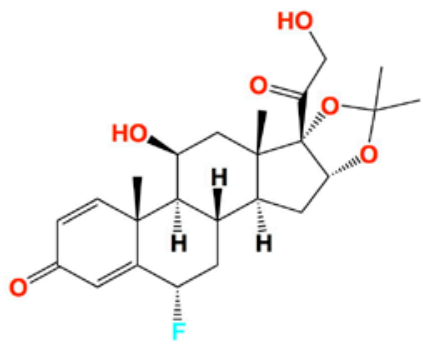

Flunisolide

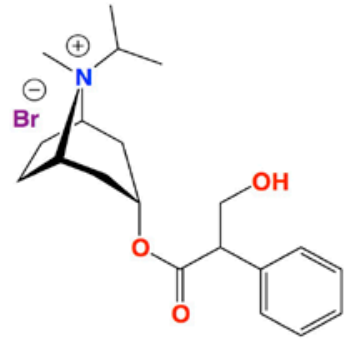

Ipratropium Bromide

Figure 1: Molecule structure for the five selected active pharmaceutical ingredients. 

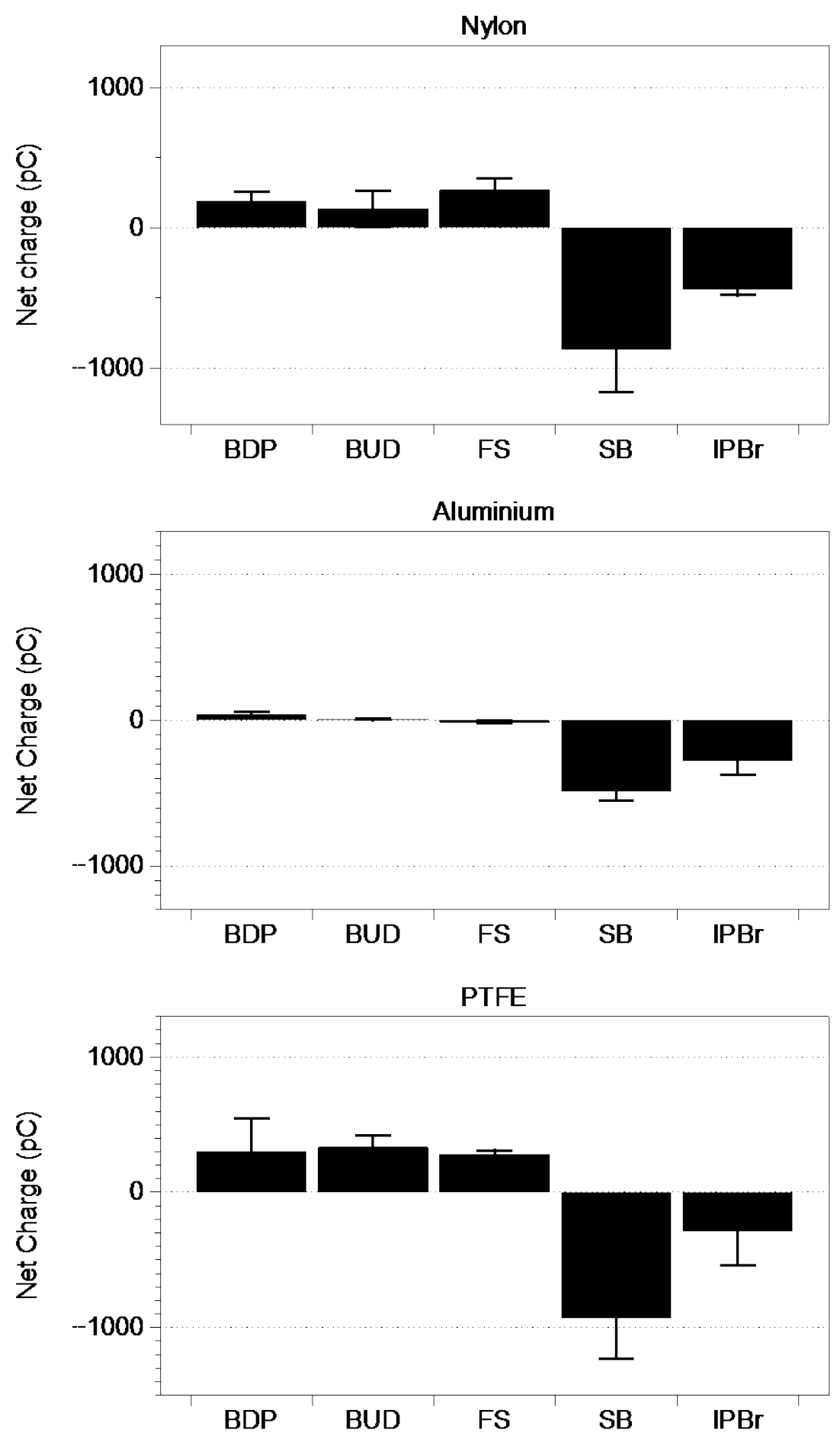

Figure 2: Net charge for all APIs with nylon, aluminium and PTFE actuators, $(\mathbf{n}=3 \pm \mathrm{SD})$ 

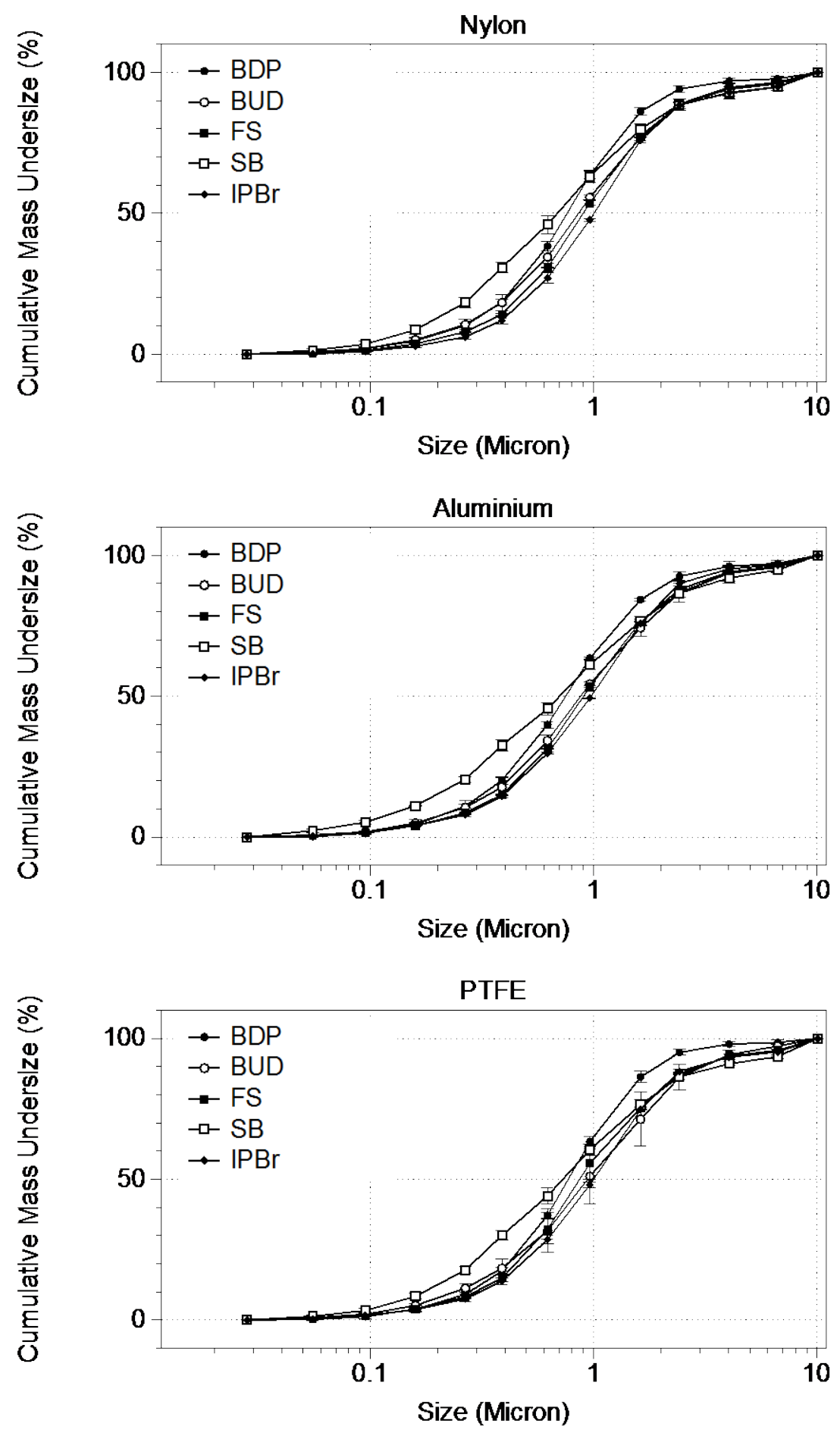

Figure 3: Cumulative mass undersize plots for all APIs with nylon, aluminium and PTFE actuators ( $n=3, \% \mathrm{CMU} \pm \mathrm{SD})$ 


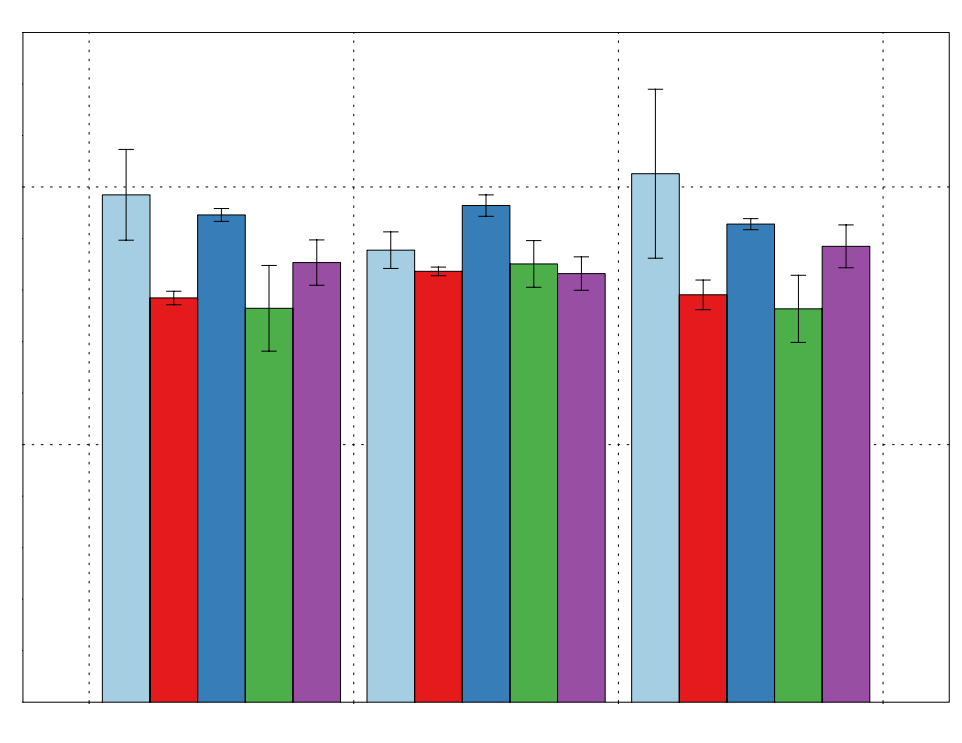

Figure 4: Total ex-valve dose for all APIs with nylon, aluminium and PTFE actuators, expressed as \% of targeted dose: 5 accumulative shots of $50 \mu \mathrm{g}$ per shot equivalent to $250 \mu \mathrm{g},(\mathrm{n}=3 \pm \mathrm{SD})$

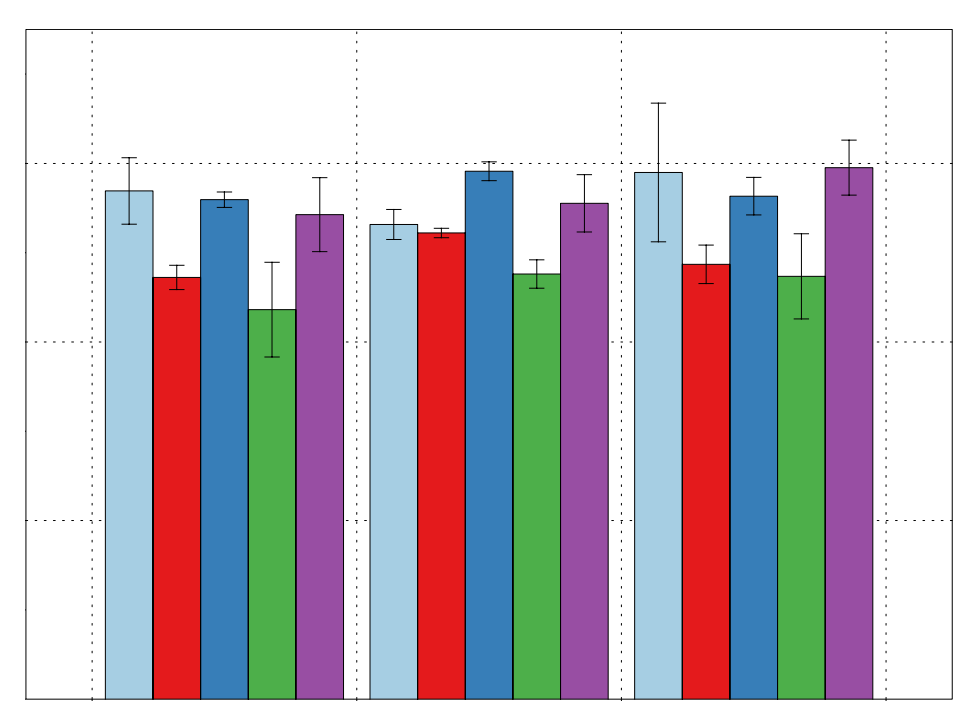

Figure 5: Throat USP deposition for all APIs with nylon, aluminium and PTFE actuators, expressed as \% of targeted dose: 5 accumulative shots of $50 \mu \mathrm{g}$ per shot equivalent to $250 \mu \mathrm{g},(\mathrm{n}=3 \pm \mathrm{SD})$ 


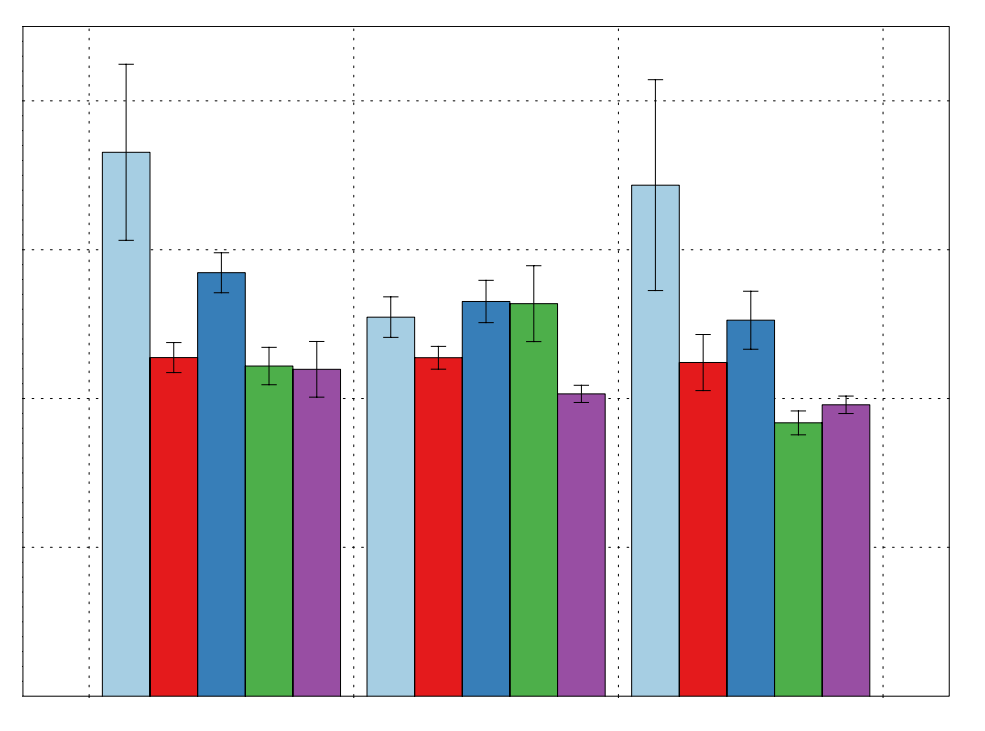

Figure 6: Fine particle fraction less than $6.66 \mu \mathrm{m}$ for all APIs with nylon, aluminium and PTFE actuators, expressed as \% of targeted dose: 5 accumulative shots of $50 \mu \mathrm{g}$ per shot equivalent to $250 \mu \mathrm{g},(\mathrm{n}=3 \pm \mathrm{SD})$ 